\title{
La question du "statut du chercheur africain" comme discours stratégique
}

Lazare Maurice Sehoueto

\section{OpenEdition}

\section{Journals}

Édition électronique

URL : http://journals.openedition.org/apad/342

DOI : 10.4000/apad.342

ISSN : 1950-6929

Éditeur

LIT Verlag

Édition imprimée

Date de publication : 15 mars 1991

\section{Référence électronique}

Lazare Maurice Sehoueto, "La question du "statut du chercheur africain" comme discours stratégique », Bulletin de l'APAD [En ligne], 1 | 1991, mis en ligne le 23 juin 2006, consulté le 08 septembre 2020. URL : http://journals.openedition.org/apad/342 ; DOI : https://doi.org/10.4000/apad. 342

Ce document a été généré automatiquement le 8 septembre 2020

Bulletin de I'APAD 


\title{
La question du "statut du chercheur africain" comme discours stratégique
}

\author{
Lazare Maurice Sehoueto
}

1 On a évoqué une prétendue "problématique du statut du chercheur africain", entre autres questions soulevées et plus ou moins discutées au cours des assises constitutives de "APAD, en la qualifiant de "cardinale". Les mauvaises solutions à elle apportées par les dirigeants africains (ces "médiocrités" qui osent déclarer qu'ils "trouvent des chercheurs qui cherchent mais point de chercheurs qui trouvent") expliqueraient pourquoi le chercheur africain reste sevré de production scientifique -les exceptions confirment la règle- puis n'arrive pas à contribuer autant qu'on pourrait s'y attendre, au développement. Cette "problématique" décomposée avec dextérité en problèmes de "libertés" et de "moyens", avait bénéficié d'une attention particulière de l'assistance, dans une atmosphère quasi-pathétique de consensus. Il me parait impératif d'opérer une distanciation par rapport à une certaine vision de la question du statut du chercheur africain qui, en dernière instance, légitime et sert de justification a posteriori à l'infantilisation de celui-ci.

Décoder notre propre discours

2 Poser le problème du "statut du chercheur africain" et le traiter d'une manière apparemment innocente, voire généreuse, ne relève pas en soi d'une originalité. Il suffit de consulter n'importe quel cahier de revendications syndicales des professeurs des Universités africaines pour s'en convaincre. Au "Premier Congrès des Hommes de Science en Afrique" (Brazzaville, 1987), cette question a su être d'une importance telle qu'elle a fait l'objet de la Recommandation $n^{\circ}$ 3. Évoquée sur divers tons dans le cadre des associations de chercheurs impliquant aussi bien ceux du Nord que ceux du Sud, la question mérite d'être analysée de plus près.

3 Autant que les "projets de développement", les Association de chercheurs tiers-mondistes ou plus restrictivement, africanistes, les institutions de recherches sur l'Afrique, puis l'histoire réelle de l'apparition, de l'organisation et de la conduite des 
projets de recherches, pourraient être analysées avec les paradigmes de l'anthropologie du changement social et du développement (Bierschenck). Cette dernière ne doit donc pas s'arrêter à la porte du panthéon des chercheurs. Elle doit y pénétrer et, par un geste délibérément iconoclaste, faire du discours et de la pratique qui fondent et accompagnent la recherche anthropologique en Afrique, un objet d'étude avec les instruments théoriques de l'anthropologie du développement. Car, faut-il y revenir, qu'ils soient Africains ou non, les anthropologues ayant l'Afrique comme champ d'investigation sont des protagonistes du développement. Ces protagonistes, d'un genre particulier, ont parfois, par rapport aux experts, assistants étrangers et fonctionnaires nationaux qu'ils ne se lassent d'observer, de tourner et de retourner dans tous les sens, une influence plus grande qu'il n'y parait sur les "politiques de développement" que mènent les gouvernements africains (parmi lesquels, pour une raison ou une autre, certains chercheurs de renom ne manquent pas de "solides relations"). Les "Pères de la Nation" ne détestent pas toujours la compagnie des africanistes célèbres et ceux-ci sont parfois assez "réalistes" pour ne pas se refuser de leur offrir ce plaisir.

4 Surtout qu'ils pourraient en profiter, pensent-ils, pour les informer de la "situation réelle" du pays "que leur cache la horde de courtisans qui les entourent". Trois autres raisons militent en faveur de la nécessité de thématiser, dans le champ de l'anthropologie du développement, l'organisation des chercheurs et des recherches africanistes.

5 a) Que les résultats de leurs recherches soient peu ou prou pris en compte par les "décideurs politiques", les anthropologues seraient les derniers à ne pas considérer leur science ou leurs activités scientifiques dans leurs liens avec la sphère du développement. Et "penser l'activité scientifique comme un moment particulier de l'activité économique au sens large, permet justement de mieux éclairer les mystères apparents de la production des connaissances en pays dominés, et des rapports de production qui les caractérisent." (Hountondji). Par ailleurs, il s'avère révélateur que l'APAD se propose dans ses statuts (Art.2 ; al.3), de "permettre une meilleure prise en compte des compétences anthropologiques dans le processus de développement".

6 b) Le regain d'intérêt pour l'anthropologie dans le secteur du développement, (Chauveau, Marzouk) implique l'anthropologue, de gré ou non, dans la pratique du développement.

7 c) La science qu'elle soit "molle" ou "dure" peut, à juste titre, être essentiellement considérée comme l'un des éléments constitutifs des forces productives d'une formation sociale. La relation entre savoir et pouvoir est devenue irréfutable et très ostensible de par l'organisation actuelle de la recherche scientifique et toute la panoplie juridique et sécuritaire qui l'accompagne dans les pays dits développés. Le savoir anthropologique reste d'autant plus incontournable que les changements sociaux les plus significatifs ne s'opèrent jamais que lorsque la société est suffisamment informée sur elle-même (Elwert).

8 Il me semble, par conséquent, d'une haute importance, dans un intérêt d'abord épistémologique, que soient décodés le discours et la pratique des "recherches africanistes" comme un aspect particulier du discours et de la pratique du développement.

L'APAD vers une structuration du clientélisme? 
Circonscrire les significations possibles de l'avènement de l'APAD présentera certes bien des difficultés. Mais pourquoi ne pas se poser ces questions, tendancieuses à volonté, quoiqu'il relèverait sans doute de la spéculation d'y répondre ici et maintenant :

- de quelle nécessité a-t-on pu tirer la conséquence d'initier une organisation (de plus ?) dans cet univers plutôt surchargé où vivote un nombre non négligeable de structures s'occupant plus ou moins des mêmes questions et souvent avec les mêmes membres?

- à quelle logique répond le profil de fourre-tout imprimé à cette association (souci d'interdisciplinarité ou d'ouverture ?) qui, déjà, aux assises constitutives, a rendu bien des quiproquos et difficultés de compréhension inévitables?

- ne peut-on pas trouver dans la naissance de l'APAD la manifestation de "l'esprit gaulliste" qui, schématisé dans ses aspects qui nous intéressent ici, signifie : maintien du rôle et des responsabilités de la France (donc des Français et du français), méfiance et défiance vis-à-vis du monde anglo-saxon, échafaudage et renforcement des liens clientélistes avec les "assimilés" ?

Reconnaître l'impossibilité de répondre de manière consistante à ces questions ne devrait pas signifier qu'il serait légitime de créer les conditions pour que l'APAD rejoigne, dans les faits les autres associations du genre, sur le point d'un mignon péché largement partagé, celui de favoriser ou de conforter l'auto-infantilisation du chercheur africain. En quoi le débat sur le "statut du chercheur africain", tel qu'il a été posé, crée-t-il de telles conditions?

La question des "libertés" et des "moyens"

11 Si la jouissance des libertés publiques constitue une condition indispensable pour la créativité et les recherches scientifiques, elle ne les détermine pas en soi, de manière absolue. L'histoire des sciences en Europe, par rapport à la question des libertés, montre bien que l'Inquisition n'était certainement pas plus clémente que nos dictatures d'aujourd'hui en Afrique. Les sociétés savantes secrètes, même au cœur de l'Église, ont joué très souvent un rôle d'autant plus utile à la recherche et au progrès scientifiques, qu'elles se sont constituées et ont travaillé par opposition à la science officielle. Peut-être aurons-nous bientôt, grâce à nos "démocratisations" en cours, l'agréable surprise de constater un "boom" impressionnante de publications ou simplement de manuscrits des chercheurs Africains.

Ici le scepticisme reste permis, car l'absence de liberté ne constituait pas à mon sens, le premier élément limitant. Il me parait plus fructueux, du point de vue de l'analyse, de remarquer que la plupart des chercheurs africains sont des fonctionnaires d'État ou d'organismes internationaux qui, souvent, attendent de leurs employeurs et des institutions qui "coopèrent" avec leurs employeurs, non seulement "les moyens" mais aussi le programme et la destination des recherches. Cette attitude façonnée à la mesure de la logique clientéliste qui préside à l'aide au développement en général (Bierschenck et Elwert) , y compris l'organisation de la recherche, bloque la prise en charge $\mathrm{du}$ chercheur africain par lui-même et lui permet de contourner ses responsabilités propres (s'il s'en reconnaissait). Il est remarquable par ailleurs que la promotion d'un chercheur africain, surtout dans les pays dits francophones, est moins liée à ses prestations et à sa compétence professionnelles qu'à son "ancienneté dans le corps". Ainsi, ni le nombre, ni la qualité des publications ou simplement des recherches, n'apparaissent ici pour déterminer le "statut du chercheur". Lorsque, dans un tel contexte, surgit de manière spéculative une "problématique du statut du 
chercheur africain" il y a d'abord un impératif de mise entre parenthèses des aspects relativement objectifs de la question puis à traiter des non-dits et des ambiguïtés qu'elle véhicule. Elle est surtout à analyser comme discours stratégique et mise en scène. Il me semble d'autant plus utile de procéder à une telle analyse que l'on oublie généralement de mentionner, par exemple, qu'en Afrique, en termes relatifs, les chercheurs et autres professeurs d'université constituent à l'intérieur de leur pays une couche sociale privilégiée dont les privilèges ne sont jamais fixés en fonction de leur productivité ou de son caractère stratégique dans la production ou la mise en valeur des richesses nationales. Un pays comme la Centrafrique ,qui tire $80 \%$ de son budget de l'aide extérieure, ne peut logiquement percevoir la recherche et la production scientifique comme un facteur économique majeur au point de "privilégier" ses chercheurs à cet effet.

Si les chercheurs africains restent cependant des privilégiés dans leur pays, c'est surtout dans le cadre d'une stratégie de conservation du pouvoir qu'appliquent les gouvernements. Aussi a-t-on pu, dans un pays comme le Bénin, arrêter comme base de rémunération le nombre d'années d'études faites après ou avant tel diplôme, puis "l'ancienneté dans la fonction". Dans un tel système (que l'on retrouve dans nombre d'autres pays africains), le chercheur qui n'est reconnu comme tel d'abord que par ce qu'il a effectué un certain nombre d'années d'études universitaires, peut en Afrique réussir l'exploit de ne rien chercher, de ne rien publier et connaître cependant de la "promotion sociale" et même se plaindre du "statut (d'enfant pauvre) du chercheur africain". Il n'est pas du tout rare de retrouver dans quelque université africaine, des "professeurs-chercheurs" qui enseignent (au sens étymologique du mot) depuis une vingtaine d'années, les conclusions de leur thèse de doctorat (brillamment soutenue en Europe !), qui encaissent par mois, en sus de leur salaire, 20 à $30 \%$ de celui-ci, en guise "d'indemnités de recherches" sans que nulle part apparaisse ne serait-ce que des résultats provisoires de leurs recherches. Leur "avancement" sur le plan professionnel ne s'en trouve pas pour autant compromis. Ainsi, les quelques "professeurs-chercheurs" qui, au Bénin par exemple, s'adonnent effectivement à la recherche, n'ont pas un "statut" meilleur que celui des autres et font presque figure de gens "anormaux".

Tant que, en effet, la question du statut du chercheur africain ne sera pas perçue en relation plus intime avec sa production et sa productivité scientifiques, dans les limites actuelles de ses moyens actuels, elle sera porteuse d'ambiguïtés nuisibles à la reconnaissance sociale d'un statut de chercheur en Afrique. En outre, elle constituera à la fois une justification de non-travail et le dernier refuge d'un clientélisme et d'un parasitisme peut-être utiles pour maintenir la dépendance de la "périphérie". 